PROCEEDINGS OF THE

AMERICAN MATHEMATICAL SOCIETY

Volume 140, Number 11, November 2012, Pages 3993-4002

S 0002-9939(2012)11250-X

Article electronically published on March 28, 2012

\title{
ENTROPY, WEIL-PETERSSON TRANSLATION DISTANCE AND GROMOV NORM FOR SURFACE AUTOMORPHISMS
}

\author{
SADAYOSHI KOJIMA
}

(Communicated by Daniel Ruberman)

\begin{abstract}
Thanks to a theorem of Brock on the comparison of Weil-Petersson translation distances and hyperbolic volumes of mapping tori for pseudoAnosovs, we prove that the entropy of a surface automorphism in general has linear bounds in terms of a Gromov norm of its mapping torus from below and an inbounded geometry case from above. We also prove that the WeilPetersson translation distance does the same from both sides in general. The proofs are in fact immediately derived from the theorem of Brock, together with some other strong theorems and small observations.
\end{abstract}

\section{INTRODUCTION}

Let $\Sigma=\Sigma_{g, n}$ be an orientable surface of genus $g$ with $n$ punctures, $\mathcal{T}=\mathcal{T}_{g, n}$ be the Teichmüller space of $\Sigma$ and $\mathcal{M}=\mathcal{M}_{g, n}$ be the (orientation preserving) mapping class group of $\Sigma$. An element of $\mathcal{T}$ is defined by a pair $(R, f)$ of a Riemann surface $R$ homeomorphic to $\Sigma$ with a marking homeomorphism $f: \Sigma \rightarrow R$. A mapping class $\varphi \in \mathcal{M}$ naturally acts on $(R, f) \in \mathcal{T}$ by $\left(R, f \circ \varphi^{-1}\right)$. Throughout this paper, we assume that $3 g-3+n \geq 1$ or $(g, n)=(0,3)$, and fix $g$ and $n$. Also, we omit a marking homeomorphism $f$ to indicate a marked Riemann surface in $\mathcal{T}$.

In this paper, we discuss three invariants measuring the complexity of a conjugacy class of a mapping class $\varphi \in \mathcal{M}$. One is the infimum of topological entropies of surface automorphisms in $\varphi$, which we denote by ent $\varphi$. As we will point out in Corollary 10, it is equal to the Teichmüller translation distance of $\varphi \in \mathcal{M}$,

$$
\|\varphi\|_{\mathrm{T}}=\inf _{R \in \mathcal{T}} d_{\mathrm{T}}(R, \varphi(R)),
$$

where $d_{\mathrm{T}}$ denotes the Teichmüller distance on $\mathcal{T}$. The second one is the WeilPetersson translation distance defined by

$$
\|\varphi\|_{\mathrm{WP}}=\inf _{R \in \mathcal{T}} d_{\mathrm{WP}}(R, \varphi(R)),
$$

where $d_{\mathrm{WP}}$ denotes the Weil-Petersson distance on $\mathcal{T}$. The last one through three dimensional manifolds is the Gromov norm $\|\cdot\|_{\text {Gr }}$ of the mapping torus of $\varphi$,

$$
N_{\varphi}=\Sigma \times[0,1] /(x, 1) \sim(\varphi(x), 0) .
$$

Received by the editors May 6, 2010 and, in revised form, March 6, 2011 and May 13, 2011.

2010 Mathematics Subject Classification. Primary 37E30; Secondary 57M27, 57M50.

Key words and phrases. Surface automorphism, entropy, Teichmüller translation distance, Weil-Petersson translation distance, Gromov norm.

The author was partially supported by Grant-in-Aid for Scientific Research (A) (No. 22244004), JSPS, Japan.

(C)2012 American Mathematical Society Reverts to public domain 28 years from publication 
Here the notation $N_{\varphi}$ indicates only a mapping class because the topological type of $N_{\varphi}$ does not depend on the choice of representatives of $\varphi$. In fact, it depends only on the conjugacy class of $\varphi$. Hence any topological invariant of $N_{\varphi}$ such as the Gromov norm is an invariant of a mapping class $\varphi$.

The starting point of this paper is a comparison of the Weil-Petersson translation distances of pseudo-Anosovs and volumes of their mapping tori by Brock in [4. We discussed similar inequalities in Theorem 3.2 of 9 , where we replaced the WeilPetersson translation distance in Brock's work by the entropy. Here we generalize it further to one for general surface automorphisms as follows.

Theorem 1. There is a constant $A=A(g, n)>0$ which depends only on the genus $g$ and the number $n$ of punctures such that the inequality

$$
A\left\|N_{\varphi}\right\|_{\mathrm{Gr}} \leq \operatorname{ent} \varphi
$$

holds for any $\varphi \in \mathcal{M}_{g, n}$. Furthermore for any $\varepsilon>0$, there is a constant $B=$ $B(g, n, \varepsilon)>0$ which depends only on $g, n$ and $\varepsilon$ such that

$$
\text { ent } \varphi \leq B\left\|N_{\varphi}\right\|_{\mathrm{Gr}}
$$

holds for any $\varphi \in \mathcal{M}_{g, n}$ of which the hyperbolic pieces of $N_{\varphi}$ contain no closed geodesics of length $<\varepsilon$.

We also generalize the theorem of Brock to one for general surface automorphisms as follows.

Theorem 2. There exists a constant $C=C(g, n)$ which depends only on $g$ and $n$ such that

$$
C^{-1}\left\|N_{\varphi}\right\|_{\mathrm{Gr}} \leq\|\varphi\|_{\mathrm{WP}} \leq C\left\|N_{\varphi}\right\|_{\mathrm{Gr}}
$$

holds for any $\varphi \in \mathcal{M}_{g, n}$.

Both theorems are immediately derived from Brock's theorem together with some existing strong theorems and small observations. We collect necessary materials first with a reasonable account of explanations as packages in the next section, and then present a reducing argument in the last section. For the reader's convenience, we duplicate some of the arguments in $[9]$.

\section{PACKAGES}

2.1. Entropy. Let us review the topological entropy defined by Adler, Konheim and McAndrew in [1] for a self-map $f: X \rightarrow X$ of a compact topological space $X$. Choose an open covering $\mathcal{U}$ of $X$, and let $N(m, \mathcal{U})$ be the minimal number of members in a refined covering $\mathcal{U} \vee f^{-1}(\mathcal{U}) \vee \cdots \vee f^{-(m-1)}(\mathcal{U})$ by intersections that cover $\Sigma$. Then

$$
h(f)=\sup _{\mathcal{U}}\left(\limsup _{m \rightarrow \infty} \frac{1}{m} \log N(m, \mathcal{U})\right)
$$

is called the topological entropy of $f$. It is shown in [1] to always exist and to satisfy the identity

$$
h\left(f^{n}\right)=n h(f)
$$

for any positive integer $n$.

Our topological space $\Sigma$ may not be compact; however, if we suppose that the covering $\mathcal{U}$ contains open sets including each puncture in their interior, then it can 
be handled as a compact space and every argument in [1] works equally well. For a mapping class $\varphi \in \mathcal{M}$, we define the entropy of $\varphi$ by

$$
\text { ent } \varphi=\inf _{f \in \varphi} h(f) \text {. }
$$

In particular, we have the identity

$$
\text { ent } \varphi^{n}=n \operatorname{ent} \varphi
$$

2.2. Thurston's classification of surface automorphisms. According to a seminal work of Thurston in [20] (see also [7]), the elements of $\mathcal{M}$ are classified into three types: periodic, pseudo-Anosov and reducible. This classification has become standard, but it is better to note that the classification is not in trichotomy. In fact, there is a class which is periodic and also reducible.

A pseudo-Anosov mapping class $\varphi$ is known to have a good deal of structures. It has a nice representative $f: \Sigma \rightarrow \Sigma$ which leaves a pair of stable and unstable foliations invariant. The expanding factor $\lambda$ of the unstable foliation is called the dilatation of $f$. Thurston [20] (see also [7) showed that if $\varphi \in \mathcal{M}$ is pseudo-Anosov, then

$$
\text { ent } \varphi=\log \lambda \text {. }
$$

2.3. Bers' classification of surface automorphisms. Bers gave an alternative proof of Thurston's classification in 22 by adopting an extremal approach in quasiconformal mappings. Since Bers' classification is more suitable for us, we briefly review it.

Bers calls $\varphi$ elliptic if $\|\varphi\|_{\mathrm{T}}=0$, and there is a Riemann surface $R \in \mathcal{T}$ such that $\varphi(R)=R$. This is identical with Thurston's periodic case. Call $\varphi$ hyperbolic if $\|\varphi\|_{\mathrm{T}}>0$, and there is a Riemann surface $R \in \mathcal{T}$ which attains the infimum. This is also identical with Thurston's pseudo-Anosov case. The remaining cases in Bers' classification are called parabolic if $\|\varphi\|_{\mathrm{T}}=0$ or pseudo-hyperbolic if $\|\varphi\|_{\mathrm{T}}>0$, where there are no Riemann surfaces in $\mathcal{T}$ which attain the infimum for either cases. These are reducible but not periodic in Thurston's classification.

Then Bers showed that if $\varphi$ is hyperbolic (= pseudo-Anosov), then there is a unique invariant Teichmüller geodesic $\ell_{\mathrm{T}}(\varphi)$ for the action of $\varphi$ on $\mathcal{T}$, and the identity

$$
\log \lambda=d_{\mathrm{T}}(R, \varphi(R))=\|\varphi\|_{\mathrm{T}}
$$

holds for any $R \in \ell_{\mathrm{T}}(\varphi)$.

2.4. Gromov norm. According to Section 6.5 of [19, we recall the definition of the Gromov norm for a 3-manifold with toral boundary. Let $M$ be a compact orientable 3-manifold whose boundary consists of tori. Then, the Gromov norm is defined to be the limit infimum of an $\ell_{1}$-norm of cycles representing the relative fundamental class $[M, \partial M] \in H_{3}(M, \partial M ; \mathbb{R})$ with vanishing boundary condition, namely,

$$
\|[M]\|_{\mathrm{Gr}}=\liminf _{\varepsilon \rightarrow 0}\left\{\|c\|_{1} ;[c]=[M, \partial M], \text { and }\|\partial c\| \leq \varepsilon\right\} .
$$

In [18, Soma proved the splitting theorem for the Gromov norm for compact orientable 3-manifolds whose boundary consists of tori. His theorem, together with the solution of the geometrization conjecture by Perelman [16, 17, establishes a simpler statement than the original in [18] as follows. Let $v_{3}=1.01494 \ldots$ be the volume of the regular ideal hyperbolic 3 -simplex. 
Theorem 3 (Soma [18). The Gromov norm of a compact orientable 3-manifold $M$ whose boundary consists of tori is equal to $v_{3}$ times the sum of volumes of hyperbolic pieces in the geometric decomposition of $M$.

Thus the term $\left\|N_{\varphi}\right\|_{\mathrm{Gr}}$ in the inequalities of the theorems can be replaced by the sum of volumes of hyperbolic parts of $N_{\varphi}$ in the geometric decomposition.

One of the useful properties of the Gromov norm for us is that if $\widetilde{M} \rightarrow M$ is an $n$-fold covering, then

$$
\|\widetilde{M}\|_{\mathrm{Gr}}=n\|M\|_{\mathrm{Gr}} .
$$

This fact will be used to reduce the problem in question in the next subsection.

2.5. Reduction. To each mapping class $\varphi \in \mathcal{M}$ is associated a reduced maximal family $\mathcal{C}$ of disjoint simple closed curves on $\Sigma$ which is invariant by the action of $\varphi$. This means more precisely that $\Sigma-\mathcal{C}$ is divided into two groups, $U$ and $V$, which consist of components of $\Sigma-\mathcal{C}$ so that $\varphi$ restricts to $U$ is periodic and to $V$ is pseudo-Anosov, where $U$ and $V$ may be disconnected.

We know from (2.1) and (2.2) that ent and \|\|$_{\text {Gr }}$ behave well under taking powers. The same identities hold for the Weil-Petersson translation distance and Teichmüller translation distance by definition; namely,

$$
\left\|\varphi^{n}\right\|_{\mathrm{WP}}=n\|\varphi\|_{\mathrm{WP}} \quad \text { and } \quad\left\|\varphi^{n}\right\|_{\mathrm{T}}=n\|\varphi\|_{\mathrm{T}}
$$

hold for any positive integer $n$. Thus, it is sufficient to show the inequalities in the theorems in the introduction for some power of $\varphi$, and we may assume by taking some power of $\varphi$ that $\varphi$ (with the same notation) preserves each component of $\Sigma-\mathcal{C}$. Then $\Sigma$ will be a union of subsurfaces $S_{1} \cup S_{2} \cup \cdots \cup S_{k}$ split by $\mathcal{C}$, and the restriction of $\varphi$ to $S_{i}$ defines a mapping class $\varphi_{i}$ on each component $S_{i}$. If some $\varphi_{i}$ is periodic, again taking further power, we may assume that it is the identity. Thus, relabeling suffices, and letting $j$ be the number of pseudo-Anosov components, we assume in the sequel that

Reduction Hypothesis 4. The restriction $\varphi_{i}$ of $\varphi$ to $S_{i}$ in $\Sigma-\mathcal{C}=S_{1} \cup S_{2} \cup \cdots \cup S_{k}$ preserves $S_{i}$ and is pseudo-Anosov if $i \leq j(\leq k)$ and the identity if $i>j$.

Note that $j$ could range from zero to $k$. Also, $k$ could be 1 .

2.6. Geometric decomposition of $N_{\varphi}$. Thurston proved in [21] (cf. [15]) that the mapping torus of a pseudo-Anosov admits a hyperbolic structure. The hyperbolic structure in dimension $\geq 3$ is known to be unique by Mostow rigidity. Under the Reduction Hypothesis 4 , the geometric decomposition of $N_{\varphi}$ is quite simple and the hyperbolic pieces consist of $N_{\varphi_{i}}$ 's for $i \leq j$. Thus by Theorem 3 of Soma ([18]), we have

$$
\left\|N_{\varphi}\right\|_{\mathrm{Gr}}=\sum_{i=1}^{j}\left\|N_{\varphi_{i}}\right\|_{\mathrm{Gr}}=\frac{1}{v_{3}} \sum_{i=1}^{j} \operatorname{vol} N_{\varphi_{i}} .
$$

2.7. Teichmüller metric. The Teichmüller distance beween two Riemann surfaces $R, R^{\prime} \in \mathcal{T}$ is originally defined by

$$
d_{\mathrm{T}}\left(R, R^{\prime}\right)=\inf _{h} \sup _{x \in R} \log K_{h}(x)
$$


where $h: R \rightarrow R^{\prime}$ is a quasiconformal map and $K_{h}(x)$ is a dilatation of $h$ at $x$. To see the infinitesimal form of $d_{\mathrm{T}}$, we recall a little analysis by quoting a few well known results from the text book by Gardiner and Lakic 8 .

Let $R$ be a Riemann surface. Also, let $T^{1,0} R$ and $T^{0,1} R$ be the holomorphic part and the anti-holomorphic part of the complex cotangent bundle over $R$ respectively. A Beltrami differential $\mu$ on $R$, which is a section of a line bundle $\left(T^{0,1} R\right) \otimes\left(T^{1,0} R\right)^{*}$, represents an infinitesimal deformation of the complex structure of $R$ including holomorphically trivial ones caused by diffeomorphisms. We let $L_{\infty}(R)$ be the space of uniformly bounded Beltrami differentials, namely $\left\{\mu:\|\mu\|_{\infty}<\infty\right\}$. This is an infinite dimensional space.

Another object of interest is a quadratic differential $q$ which is a section of a line bundle $\left(T^{1,0}\right)^{\otimes 2}$. Let $Q(R)$ be the space of holomorphic quadratic differentials with bounded $L_{1}$ norm $\|q\|=\int_{R}|q|<\infty$. By Riemann-Roch, the dimension of $Q(R)$ is equal to $3 g-3+n$. Then, there is a natural pairing defined by

$$
(\mu, q)=\int_{R} \mu q
$$

If we let

$$
N=\{\mu:(\mu, q)=0 \text { for all } q \in Q(R)\},
$$

then $L_{\infty}(R) / N$ can be identified with the tangent space $T_{R} \mathcal{T}$ of $\mathcal{T}$ at $R$. A standard argument in functional analysis implies that

Lemma 5 (cf. 3.1, Theorem 2 in [8]). The natural pairing (2.4) induces an isomorphism of $Q(R)^{*}$ to $L_{\infty}(R) / N \cong T_{R} \mathcal{T}$.

Lemma 6 (cf. 4.12 Theorem 13 in [8]). The norm $\|\mu\|=\sup \{(\mu, q) ;\|q\| \leq 1\}$ dual to the $L_{1}$ norm on $Q(R) \cong T_{R}^{*} \mathcal{T}$ is the infinitesimal form of the Teichmüller distance $d_{\mathrm{T}}$.

2.8. Weil-Petersson metric. The Weil-Petersson metric comes from the $L^{2}$ inner product on $Q(R) \cong T_{R}^{*} \mathcal{T}$ defined by

$$
\left\langle q, q^{\prime}\right\rangle_{\mathrm{WP}}=\int_{X} \frac{\bar{q} q^{\prime}}{\rho^{2}}
$$

where $q, q^{\prime} \in Q(R)$ and $\rho(z)|d z|$ is the hyperbolic metric on $\Sigma$ conformally equivalent to the complex structure on $R$. The Weil-Petersson metric is defined to be a Riemannian part of the dual Hermitian metric to the above co-metric on the cotangent space.

The Weil-Petersson metric is known to have negatively curved sectional curvature by the work of several authors; see for example Wolpert [23]. Also, it is known to be incomplete by Wolpert in [22] but geodesically convex also by Wolpert in 24]: for each pair of points, there exists a unique distance realizing joining curve.

Daskalopoulis and Wentworth [6] showed that if $\varphi$ itself is pseudo-Anosov, then it admits the unique invariant Weil-Petersson geodesic $\ell_{\mathrm{WT}}(\varphi)$ of the action by $\varphi$ on $\mathcal{T}$, and the identity

$$
d_{\mathrm{WP}}(R, \varphi(R))=\|\varphi\|_{\mathrm{WP}}
$$

holds for any $R \in \ell_{\mathrm{WP}}(\varphi)$. 
2.9. Teichmüller versus Weil-Petersson. Lemma [6 says that the $L_{1}$ norm on $Q(R)$ is the dual norm to the infinitesimal form of the Teichmüller distance through the natural paring established in Lemma 5. Then, by the Cauchy-Schwarz inequality, we have

$$
\|q\|^{2}=\left(\int_{R}|q|\right)^{2}=\left(\int_{R} \rho \cdot \frac{|q|}{\rho}\right)^{2} \leq \int_{R} \rho^{2} \cdot \int_{R} \frac{\bar{q} q}{\rho^{2}}=\text { Area } \Sigma \cdot\langle q, q\rangle_{\mathrm{WP}} .
$$

Thus, this infinitesimal inequality on the dual space implies the inequality of the other direction in two distances,

$$
(\sqrt{\operatorname{Area} \Sigma})^{-1} d_{\mathrm{WP}} \leq d_{\mathrm{T}}
$$

which was originally proved by Linch in 10 . This implies

Lemma 7. There is a constant $D=D(g, n)$ which depends only on $g, n$ such that the inequality

$$
\|\varphi\|_{\mathrm{WP}} \leq D\|\varphi\|_{\mathrm{T}}
$$

holds for any $\varphi \in \mathcal{M}$.

Proof. Choose a Riemann surface $R$ on the Teichmüller geodesic $\ell_{\mathrm{T}}(\varphi)$ of $\varphi$ in $\mathcal{T}$. Then, we have

$$
\|\varphi\|_{\mathrm{T}}=d_{\mathrm{T}}(R, \varphi(R)) \geq(\sqrt{\operatorname{Area} \Sigma})^{-1} d_{\mathrm{WP}}(R, \varphi(R)) \geq(\sqrt{\operatorname{Area} \Sigma})^{-1}\|\varphi\|_{\mathrm{WP}},
$$

where the last inequality holds because $R$ may not be on $\ell_{\mathrm{WP}}(\varphi)$.

On the other hand, since the Weil-Petersson metric is incomplete and there is a point at infinity which is of a finite distance from an interior point, the inequality of the other direction to (2.5) cannot be established in general. However, there is such a case under some bounded geometry condition. For any $\varepsilon>0$, there is a constant $\delta>0$ such that if $\varphi \in \mathcal{M}$ is pseudo-Anosov and if a hyperbolic structure on $N_{\varphi}$ contains no closed geodesics of length $<\varepsilon$, then both the Teichmüller geodesic $\ell_{\mathrm{T}}(\varphi)$ and the Weil-Petersson geodesic $\ell_{\mathrm{WP}}(\varphi)$ are located in the subset $\mathcal{T}^{\delta}$ of $\mathcal{T}$ consisting of hyperbolic surfaces with no closed geodesics of length $<\delta$. This fact is proved for the Teichmüller metric by Minsky in 13 and for the Weil-Petersson metric by Brock-Masur-Minsky in [5]. This implies

Lemma 8. For any $\varepsilon>0$, there is a constant $E=E(g, n, \varepsilon)>0$ such that

$$
E\|\varphi\|_{\mathrm{T}} \leq\|\varphi\|_{\mathrm{WP}}
$$

for any pseudo-Anosov $\varphi \in \mathcal{M}$ so that $N_{\varphi}$ contains no closed geodesics of length $<\varepsilon$.

Proof. The subset $\mathcal{T}^{\delta}$ is invariant by the action of $\mathcal{M}$, and the quotient $\mathcal{T}^{\delta} / \mathcal{M}$ is compact by a theorem of Mumford in 14. Hence both Teichmüller and WeiPetersson metrics on $\mathcal{T}^{\delta}$ are pullbacks of metrics on the compact space $\mathcal{T}^{\delta} / \mathcal{M}$, and there is a constant $E=E(g, n, \varepsilon)$ which depend only on $g, n$ and $\varepsilon$ such that

$$
E d_{\mathrm{T}} \leq d_{\mathrm{WP}}
$$

on $\mathcal{T}^{\delta}$. Choose a Riemann surface $R$ on the Weil-Petersson geodesic $\ell_{\mathrm{WP}}(\varphi)$ of $\varphi$ on $\mathcal{T}$. Then

$$
\|\varphi\|_{\mathrm{WP}}=d_{\mathrm{WP}}(R, \varphi(R)) \geq E d_{\mathrm{T}}(R, \varphi(R)) \geq E\|\varphi\|_{\mathrm{T}},
$$

where the last inequality holds because $R$ may not be on $\ell_{\mathrm{T}}(\varphi)$. 
2.10. Weil-Petersson translation distance. The augmented structure of the completion $\overline{\mathcal{T}}$ with respect to the Weil-Petersson metric was extensively studied by Masur in [11. Using this, together with Wolpert's convexity, Masur and Wolf provided a very clear geodesically embedded picture of the frontier of $\overline{\mathcal{T}}$ in Subsection 1.2 of 12 .

One important observation there is that the intrinsic Weil-Petersson metric defined on the frontier of $\mathcal{T}$ is identical with the induced metric on the metric completion of $\mathcal{T}$ with respect to the Weil-Petersson metric. In particular, if we let $\mathcal{O}(\mathcal{C})$ be the product $\mathcal{T}\left(S_{1}\right) \times \mathcal{T}\left(S_{2}\right) \times \cdots \times \mathcal{T}\left(S_{k}\right)$ of Teichmüller spaces of $S_{i}$ 's, together with the product metric of the Weil-Petersson metrics on each Teichmüller space $\mathcal{T}\left(S_{i}\right)$, then $\mathcal{O}(\mathcal{C})$ is isometrically embedded in $\overline{\mathcal{T}}$ as the corresponding frontier of $\mathcal{T}$.

Recall that Daskalopoulis and Wentworth [6] established the existence of a WeilPetersson geodesic for the action of a pseudo-Anosov $\varphi$ on $\mathcal{T}$. The following lemma concerns the other case, which was discussed earlier in [25. We here provide its quick proof for the reader's convenience.

Lemma 9. The following hold:

(1) If $j=0$, then $\varphi$ fixes $\mathcal{O}(\mathcal{C})$ and $\|\varphi\|_{\mathrm{WP}}=0$.

(2) If $k \geq 2$ and $1 \leq j \leq k$, then there is no invariant geodesic of the action by $\varphi$ in $\mathcal{T}$, but there is at least one in $\mathcal{O}(\mathcal{C}) \subset \overline{\mathcal{T}}$, and

$$
\|\varphi\|_{\mathrm{WP}}=\sqrt{\sum_{i=1}^{j}\left\|\varphi_{i}\right\|_{\mathrm{WP}}^{2}} .
$$

The invariant geodesic is not unique if and only if $j<k$ and there exist $i>j$ so that $S_{i}$ is not a thrice punctured sphere.

Proof. (1) is clear.

To see (2), choose a point $R_{i} \in \mathcal{T}\left(S_{i}\right)$ on the invariant geodesic of the action by $\varphi_{i}$ for $i \leq j$ and any point $R_{i} \in \mathcal{T}\left(S_{i}\right)$ if $i>j$. Then the point $R=\left(R_{1}, R_{2}, \cdots, R_{k}\right)$ on $\mathcal{O}(\mathcal{C})$ will be on the geodesic $\ell$ in $\mathcal{O}(\mathcal{C}) \subset \overline{\mathcal{T}}$ invariant by the action of $\varphi$. The identity (2.7) will be obvious by the structure of the metric on $\mathcal{O}(\mathcal{C})$.

To see the non-existence of an invariant geodesic of the action by $\varphi$ in $\mathcal{T}$, suppose to the contrary that we have one $\ell^{\prime}$ lying in $\mathcal{T}$. Since $\mathcal{T}$ with the Weil-Petersson metric has negative sectional curvature and is geodesically convex, it is a CAT(0) space. Then by Chapter II, Corollary 3.11 in [3], the metric completion $\overline{\mathcal{T}}$ is also a $\operatorname{CAT}(0)$ space. Since $\ell$ and $\ell^{\prime}$ are both invariant by the action of $\varphi$, it is easy to see that they provide asymptotic geodesics, namely, there are a constant $K$ and arclength parameterizations for $\ell$ and $\ell^{\prime}$ so that $d_{\mathrm{WP}}\left(\ell(r), \ell^{\prime}(t)\right)<K$ for all $t \in \mathbb{R}$. This contradicts the flat strip theorem, Chapter II, Theorem 2.13 in $\left[\underline{3}\right.$, since $\ell^{\prime}$ is located in the part with negative sectional curvature.

2.11. Teichmüller translation distance versus entropy. By applying Theorem 4 in [1] to our setting, we obtain the identity,

$$
\operatorname{ent} \varphi=\max _{1 \leq i \leq j} \operatorname{ent} \varphi_{i} .
$$

This is the only result in this subsection that we will use in the next section. However, we would like to add a few more lines that are worth noting. 
Theorem 9 in 2] can be simply stated in our setting by

$$
\|\varphi\|_{\mathrm{T}}=\max _{1 \leq i \leq j}\left\|\varphi_{i}\right\|_{\mathrm{T}} .
$$

Since $\varphi_{i}$ is pseudo-Anosov for $i \leq j$, ent $\varphi_{i}=\left\|\varphi_{i}\right\|_{\mathrm{T}}$ holds for $i \leq j$, and we have the following corollary,

Corollary 10. The identity

$$
\operatorname{ent} \varphi=\|\varphi\|_{\mathrm{T}}
$$

holds for any $\varphi \in \mathcal{M}$.

This should be well known to the experts; however, it seems to have not been in the literature so far.

\section{Proofs}

3.1. Setting up. If $j=0$, then the entropy of $\varphi$, the Weil-Petersson translation distance of $\varphi$ and the Gromov norm of $N_{\varphi}$ all are obviously zero, and we are done for both theorems. Thus assume that $j \geq 1$. Then by the theorem of Brock in [4, we have a set of inequalities,

$$
C_{i}^{-1}\left\|\varphi_{i}\right\|_{\mathrm{WP}} \leq \operatorname{vol} N_{\varphi_{i}} \leq C_{i}\left\|\varphi_{i}\right\|_{\mathrm{WP}}, \quad i=1,2, \cdots, j .
$$

Each $C_{i}$ depends only on the topology of the surface $S_{i}$. Now, there are only finitely many topologies which can appear for surfaces obtained by splitting $\Sigma$ along a system of essential curves. Thus, there is a constant $F_{1}=F_{1}(g, n)$ which bounds the Brock constant for a surface appearing in any essential splitting of $\Sigma$. In particular, $F_{1}$ depends only on $g, n$ and does not depend on any particular splitting of $\Sigma$. Replacing $C_{i}$ by $F_{1}$ in (3.1), summing all inequalities in (3.1), and replacing the middle term by the identity (2.3), we obtain

$$
F_{1}^{-1} \sum_{i=1}^{j}\left\|\varphi_{i}\right\|_{\mathrm{WP}} \leq v_{3}\left\|N_{\varphi}\right\|_{\mathrm{Gr}} \leq F_{1} \sum_{i=1}^{j}\left\|\varphi_{i}\right\|_{\mathrm{WP}} .
$$

3.2. Proof of Theorem 1 , By Lemma 7 and Lemma 8 , we have a set of inequalities

$$
E_{i} \operatorname{ent} \varphi_{i}=E_{i}\left\|\varphi_{i}\right\|_{\mathrm{T}} \leq\left\|\varphi_{i}\right\|_{\mathrm{WP}} \leq D_{i}\left\|\varphi_{i}\right\|_{\mathrm{T}}=D_{i} \text { ent } \varphi_{i} \quad i=1,2, \cdots, j .
$$

By the same reasoning as in subsection 3.1, we can find constants $F_{2}=F_{2}(g, n, \varepsilon)$ and $F_{3}=F_{3}(g, n)$ which do not depend on any splitting of $\Sigma$ such that the equalities

$$
F_{2} \sum_{i=1}^{j} \operatorname{ent} \varphi_{i} \leq \sum_{i=1}^{j}\left\|\varphi_{i}\right\|_{\mathrm{WP}} \leq F_{3} \sum_{i=1}^{j} \operatorname{ent} \varphi_{i}
$$

hold. The left inequality is established only under the bounded geometry condition controlled by $\varepsilon$.

On the other hand, since $j$, which is the number of pseudo-Anosov components, is at most $n+2 g-2$, there is an obvious constant $F_{4}=F_{4}(g, n)>0$ which depends only on $g, n$ such that the inequalities

$$
F_{4}^{-1} \max _{1 \leq i \leq j} \operatorname{ent} \varphi_{i} \leq \sum_{i=1}^{j} \operatorname{ent} \varphi_{i} \leq F_{4} \max _{1 \leq i \leq j} \operatorname{ent} \varphi_{i}
$$


hold. Thus, by letting $F_{5}=F_{2} F_{4}^{-1}$ and $F_{6}=F_{3} F_{4}$, we have

$$
F_{5} \operatorname{ent} \varphi \leq \sum_{i=1}^{j}\left\|\varphi_{i}\right\|_{\mathrm{WP}} \leq F_{6} \operatorname{ent} \varphi
$$

Theorem 1 is immediate from the comparisons of (3.2) and (3.4).

3.3. Proof of Theorem 2, Again since $j \leq n+2 g-2$, there is an obvious constant $F_{7}=F_{7}(g, n)$ which does not depend on any splitting of $\Sigma$ such that

$$
F_{7}^{-1} \sqrt{\sum_{i=1}^{j}\left\|\varphi_{i}\right\|_{\mathrm{WP}}^{2}} \leq \sum_{i=1}^{j}\left\|\varphi_{i}\right\|_{\mathrm{WP}} \leq F_{7} \sqrt{\sum_{i=1}^{j}\left\|\varphi_{i}\right\|_{\mathrm{WP}}^{2}} .
$$

This, together with the identity (2.7) and the comparison (3.2), immediately implies Theorem 2

\section{ACKNOWLEDGMENTS}

The author would like to thank Eiko Kin and Mitsuhiko Takasawa for their helpful comments.

\section{REFERENCES}

[1] R. L. Adler, A. G. Konheim and M. H. McAndrew, Topological entropy, Trans. Amer. Math. Soc., 114 (1965), 309-319. MR0175106 (30:5291)

[2] L. Bers, An extremal problem for quasiconformal mappings and a theorem by Thurston, Acta. Math., 141 (1978), 73-98. MR0477161 (57:16704)

[3] M. Bridson and A. Haefliger, Metric spaces of non-positive curvature, Comprehensive Studies in Math., vol. 319, Springer (1999). MR1744486 (2000k:53038)

[4] J. Brock, Weil-Petersson translation distance and volumes of mapping tori, Communications in Analysis and Geometry, 11 (2003), 987-999. MR2032506 (2004k:32018)

[5] J. Brock, H. Masur and Y. Minsky, Asymptotics of Weil-Petersson geodesics II: Bounded geometry and unbounded entropy, arXiv:math.GT/1004.4401.

[6] G. Daskalopoulis and R. Wentworth, Classification of Weil-Petersson isometries, Amer. J. Math., 125 (2003), 941-975. MR.1993745(2004d:32011)

[7] A. Fathi, F. Laudenbach and V. Poenaru, Travaux de Thurston sur les surfaces, Asterisque, 66-67, Société Mathématique de France, Paris (1979). MR568308 (82m:57003)

[8] F. Gardiner and N. Lakic, Quasiconformal Teichmüller Theory, Mathematical Surveys and Monographs, Volume 76, Amer. Math. Soc. (2000). MR1730906 (2001d:32016)

[9] E. Kin, S. Kojima and M. Takasawa, Entropy versus volume for pseudo-Anosovs, Experimental Math., 18 (2009), 397-407. MR 2583541(2010j:37064)

[10] M. Linch, A comparison of metrics on Teichmüller space, Proc. Amer. Math. Soc., 43 (1974), 349-352. MR0338453 (49:3217)

[11] H. Masur, The existence of the Weil-Petersson metric to the boundary of Teichmüller space, Duke Math. J., 43 (1976), 623-635. MR0417456 (54:5506)

[12] H. Masur and M. Wolf, The Weil-Petersson isometry group, Geometriae Dedicata, 93 (2002), 177-190. MR1934697 (2003j:32017)

[13] Y. Minsky, Teichmüller geodesics and ends of hyperbolic 3-manifolds, Topology, 32 (1993), 625-647. MR:1231968 (95g:57031)

[14] D. Mumford, A remark on Mahler's compactness theorem, Proc. Amer. Math. Soc., 28 (1971), 289-294. MR0276410 (43:2157)

[15] J.-P. Otal and L. Kay, The hyperbolization theorem for fibered 3-manifolds, SMF/AMS Texts and Monographs, 7, American Mathematical Society (2001). MR:1855976 (2002g:57035)

[16] G. Perelman, The entropy formula for the Ricci flow and its geometric applications, arXiv:math.DG/0211159.

[17] G. Perelman, Ricci flow with surgery of three-manifolds, arXiv:math.DG/0303109. 
[18] T. Soma, The Gromov invariant of links, Inventiones Math., 64 (1981), 445-454. MR632984 (83a:57014)

[19] W. Thurston, The geometry and topology of 3-manifolds, Lecture Notes, Princeton University (1979).

[20] W. Thurston, On the geometry and dynamics of diffeomorphisms of surfaces, Bull. Amer. Math. Soc., 19 (1988), 417-431. MR956596 (89k:57023)

[21] W. Thurston, Hyperbolic structures on 3-manifolds II: Surface groups and 3-manifolds which fiber over the circle, preprint.

[22] S. Wolpert, Noncompleteness of the Weil-Petersson metric for Teichmüller space, Pacific J. Math., 61 (1975), 573-577. MR 0422692 (54:10678)

[23] S. Wolpert, Chern forms and the Riemann tensor for the moduli space of curves, Inventiones Math., 85 (1986), 119-145. MR842050 (87j:32070)

[24] S. Wolpert, Geodesic length functions and the Nielsen problem, J. Differential Geom., 25 (1987), 275-295. MR880186 (88e:32032)

[25] S. Wolpert, Geometry of the Weil-Petersson completion of Teichmüller space, Surveys in Differential Geometry, vol. VIII (2003), 357-393. MR2039996 (2005h:32032)

Department of Mathematical and Computing Sciences, Tokyo Institute of Technology, Ohokayama, Meguro, Tokyo 152-8552, Japan

E-mail address: sadayosi@is.titech.ac.jp 